\title{
Genetic analysis by targeted next-generation sequencing and novel variation identification of maple syrup urine disease
}

\section{Xiaohua Fang}

First Affiliated Hospital of Zhengzhou University

\section{Xiaofan Zhu}

First Affiliated Hospital of Zhengzhou University

\section{Yin Feng}

First Affiliated Hospital of Zhengzhou University

\section{Ying Bai}

First Affiliated Hospital of Zhengzhou University

\section{Xuechao Zhao}

First Affiliated Hospital of Zhengzhou University

Xiangdong Kong ( $\square$ kongxd@263.net)

First Affiliated Hospital of Zhengzhou University

Ning Liu

First Affiliated Hospital of Zhengzhou University

\section{Research Article}

Keywords: MSUD, BCAAs, BCKDHA, BCDKHB, DBT, DLD, Computational structural

Posted Date: February 24th, 2021

DOl: https://doi.org/10.21203/rs.3.rs-221149/v1

License: (c) (1) This work is licensed under a Creative Commons Attribution 4.0 International License. Read Full License 
Genetic analysis by targeted next-generation sequencing and novel variation identification of maple syrup urine disease

Xiaohua Fang, Xiaofan Zhu, Yin Feng, Ying Bai, Xuechao Zhao, Xiangdong Kong, Ning Liu. Obstetrics and Gynecology Department, Genetic and Prenatal Diagnosis Center, The First Affiliated Hospital of Zhengzhou University, 450052 P. R.China

Corresponding author: Xiangdong Kong, Email: kongxd@263.net

Ning Liu, E-mail: liuning903@163.com

\begin{abstract}
Maple syrup urine disease (MSUD) is a rare autosomal recessive disorder that affects the degradation of branched chain amino acids (BCAAs). Only a few cases of MSUD have been documented in Mainland China. In this report, 8 patients ( 4 girls and 4 boys) with MSUD from 8 unrelated Chinese families were diagnosed at the age of 9 days to 1 year and 8 months. All the coding regions and exon/intron boundaries of $B C K D H A, B C D K H B, D B T, D L D$ genes were analyzed by targeted NGS in the 8 MSUD pedigrees. Targeted NGS revealed 2 pedigrees with MSUD Ia, 5 pedigrees with Ib, 1 pedigree with MSUD II. Totally, 13 variants were detected, including 2 variants (p.Ala216Val and p.Gly281Arg) in BCKDHA gene, 10 variants (p.Gly95Ala, p.Ser171Pro, p.Phe175Leu, p.Arg183Trp, p.Lys222Thr, p.Arg285Ter, p.Arg111Ter, p.S184Pfs*46, p.Arg170Cys, p.I160Ffs*25) in $B C K D H B$ gene, 1 variants (p.Arg431Ter) in $D B T$ gene. In addition, 4 previously unidentified variants (p.Gly281Arg in BCKDHA gene, p.Ser171Pro, p.Gly95Ala and p.Lys222Thr in $B C K D H B$ gene) were found. NGS plus Sanger sequencing detection is effective and accurate for making gene diagnosis. Computational structural modeling indicated that these novel variations might affect structural stability.
\end{abstract}

\title{
Introduction
}

Maple syrup urine disease (MSUD, OMIM \# 248600) is a hereditary branched-chain amino acid metabolism disorder caused by branched chain $\alpha$-ketoacid dehydrogenase multi-enzyme complex (BCKDC). Common clinical manifestations of MSUD are feeding difficulties, epilepsy, mental retardation, ketonuria and maple-like body odor. Without timely intervention, the disease progresses rapidly, and the mortality and disability rate are very high. According to the phenotype, MSUD can be divided into 5 type $^{1}$ : classic, intermediate, intermittent, thiamine-reactive and dihydrolipoylamide dehydrogenase (E3) deficiency type. The classic type is the most common and 
severe type in the neonatal period, accounting for $75 \%$ affected infants. It usually occurs $4-9$ days after birth. The liver BCKDC activity in classic type children is often lower than $2 \%$ in healthy children, manifested as ketoacidosis, neurologic damage and mental retardation ${ }^{2} .20 \%$ are intermediate or intermittent type, the intermediate type usually shows a continuously increased concentration of branched-chain amino acids (BCAAs), accompanied by nervous system damage ${ }^{3}$. Intermittent type usually occurs from 5 months to 2 years with mild symptoms ${ }^{4}$, prognosis of the thiamine-responsive type is better than classic type, with BCKDA activity of $2 \%-40 \%$, and some children can survive for a long time ${ }^{5}$. The activity of dihydrolipoamide acyl dehydrogenase (E3) -deficient BCKDA is $25 \%$ lower than that of normal children, and it is characterized by low tension, stunting, and lactic acidosis ${ }^{6}$.

MSUD is inherited in autosomal recessive pattern, and it is very rare in most populations, with an incidence of $1: 185000^{7}$. BCKDC is located in the mitochondrial inner membrane and consists of 4 subunits Ela, E1 $\beta, \mathrm{E} 2, \mathrm{E} 3$, which are encoded by $B C K D H A, B C K D H B, D B T$ and $D L D$ genes, respectively ${ }^{8}$. According to the involved subunit, MSUD is divided into the following types: (1) type Ia (OMIM 608348), caused by biallelic pathogenic variants in BCKDHA gene encoding the Ela subunit; (2) type I b (OMIM 248611), caused by biallelic pathogenic variants in $B C K D H B$ gene encoding the E1 $\beta$ subunit; (3) type II (OMIM 248610), caused by biallelic pathogenic variants in $D B T$ gene encoding E2 subunit; (4) type III (OMIM 238331), caused by biallelic pathogenic variants in $D L D$ gene encoding E3 subunit ${ }^{7}$. Another two subtypes Type IV and type $\mathrm{V}$ are specific kinase and phosphatase gene mutation types, respectively.

MSUD is a genetically heterogeneous disease, and the traditional sequencing technology is time-consuming and costly. High-throughput sequencing technology based on target gene capture for sequencing of the four genes can simultaneously detect gene mutations in the causative genes, not only providing accurate genetic diagnosis results for patients, but also providing clinicians with the basis for differential diagnosis, drug treatment and subsequent genetic counseling, and prenatal diagnosis. In this study, we applied targeted high-throughput sequencing to sequence the target regions of $B C K D H A, B C K D H B, D B T$ and $D L D$ genes in peripheral blood samples of patients or parents in 8 families with MSUD, and Sanger sequencing validation was subsequently performed for confirmation of suspected pathogenic variants.

\section{Methods}


Subjects. Eight unrelated families that had given birth to children affected with MSUD were collected. All children in the 8 families were screened by tandem mass spectrometry and received positive screening results. Only children in family 3 and 7 accepted timely diagnosis and treatment after neonatal screening. As is shown in Table 1, The remaining 6 children developed the disease from 2 days to 2 months, and died at 16 days, 2 months, 20 days, 1 month, 10 days and 1 month, respectively. Written informed consent was obtained from the legal guardians. All of the procedures and informed consent were approved by the Medical Ethics Committee of the First Affiliated Hospital of Zhengzhou University, and was performed according to the principles of the Declaration of Helsinki.

Table 1 Clinical and laboratory features of the 8 patients with MUSD

\begin{tabular}{|c|c|c|c|c|c|c|c|c|}
\hline Patients & 1 & 2 & 3 & 4 & 5 & 6 & 7 & 8 \\
\hline Sex & $\mathrm{F}$ & $\mathrm{F}$ & $\mathrm{M}$ & $\mathrm{F}$ & $\mathrm{M}$ & $\mathrm{M}$ & $\mathrm{F}$ & $\mathrm{M}$ \\
\hline Age of Onset & $7 \mathrm{~d}$ & $1 \mathrm{~m}$ & $4 \mathrm{~m}$ & $10 \mathrm{~d}$ & $2 d$ & $3 d$ & $17 \mathrm{~d}$ & $5 d$ \\
\hline \multicolumn{9}{|l|}{ Clinical manifestation } \\
\hline Vomiting & + & - & - & + & - & - & - & - \\
\hline Poor response & + & - & - & - & + & + & + & + \\
\hline Coma & + & - & - & + & - & - & - & - \\
\hline Convulsion & + & + & - & - & - & + & + & + \\
\hline Feed difficulties & - & + & - & + & + & + & + & + \\
\hline Hypermyotonia & - & + & - & - & - & + & + & - \\
\hline Maple syrup odor & - & + & - & - & - & + & - & - \\
\hline Mental retardation & - & - & + & - & - & - & - & - \\
\hline Motor development delay & - & - & + & - & - & - & - & - \\
\hline Seizure & - & - & - & - & + & - & - & - \\
\hline Cranial CT/MRI & / & $\begin{array}{l}\text { Extensive } \\
\text { Low-densi } \\
\text { ty changes } \\
\text { in the } \\
\text { brain }\end{array}$ & $\begin{array}{l}\text { Bilateral } \\
\text { Frontotempo } \\
\text { ral parietal } \\
\text { white mater } \\
\text { density } \\
\text { symmetry } \\
\text { decreased }\end{array}$ & / & 1 & / & $\begin{array}{l}\text { Poor } \\
\text { myelination } \\
\text { in white } \\
\text { matter, } \\
\text { diffuse long } \\
\text { T1 and long } \\
\text { T2 Edema } \\
\text { signal } \\
\text { changes in } \\
\text { subcortical } \\
\text { white matter } \\
\text { and } \\
\text { frontotempor } \\
\text { al white } \\
\text { matter }\end{array}$ & / \\
\hline \multicolumn{9}{|c|}{ Blood tandem mass spectrometry } \\
\hline $\begin{array}{l}\text { Leucine/Isoleucin } \\
(\mu \mathrm{mol} / \mathrm{L})\end{array}$ & $\begin{array}{l}1904 \\
(<295)\end{array}$ & $\begin{array}{l}935 \\
(<200)\end{array}$ & $\begin{array}{r}1122.88 \\
(<375)\end{array}$ & $679(<230)$ & $3089(<375)$ & $3209(<200)$ & $\begin{array}{l}1754 \\
(<200)\end{array}$ & $2748(<300)$ \\
\hline Valine & $354(<270)$ & $\begin{array}{l}295 \\
(<210)\end{array}$ & $\begin{array}{l}325.05 \\
(<325)\end{array}$ & $420(<230)$ & $578(<325)$ & $521(<280)$ & $\begin{array}{l}302.92 \\
(<280)\end{array}$ & $520(<300)$ \\
\hline Leucine/Phenylalanine & $34.2(<7)$ & $19.4(<8)$ & $29.92(<8)$ & $12.6(<7)$ & $38.2(<8)$ & $39.1(<8)$ & $22(<8)$ & $29.5(<8)$ \\
\hline Clinical phenotype & Classic & ITD & ITD & ITD & Classic & Classic & ITD & Classic \\
\hline outcome & Died at $16 d$ & Died at $2 \mathrm{~m}$ & $\begin{array}{c}\text { Mental } \\
\text { retardation }\end{array}$ & Died at $20 \mathrm{~d}$ & Died at $1 \mathrm{~m}$ & Died at $10 \mathrm{~d}$ & $\begin{array}{l}\text { Mental } \\
\text { retardation }\end{array}$ & Died at $1 \mathrm{~m}$ \\
\hline
\end{tabular}


Notes: $F=$ Female, $M=$ Male, $\mathbf{d}=$ day, $\mathbf{m}=$ month, ITD $=$ Intermediate, ITM =Intermittent

Blood amino acid and ester Acylcarnitine Spectra analysis. Venous blood was collected from the children on an empty stomach for more than 4 hours. Blood amino acid and ester-acylcarnitine profiling was performed using liquid-tandem mass spectrometry.

DNA extraction. Blood samples $(2 \mathrm{ml})$ were collected from each patient and their parents in families 3 and 7 by venipuncture in EDTA tubes. For the remaining six families, parental blood samples were collected. Genomic DNA was extracted from peripheral blood leukocytes using a QIAamp ${ }^{\circledR}$ DNA Blood Mini Kit (Qiagen, Cat.No.51106, Germany) according to the recommended protocol.

Next-generation sequencing. Using exon capture microarrays, metabolic disease gene panel was selected for high-throughput sequencing detection, with an average sequencing depth of $200 \times$ and more than $97 \%$ of coverage at a depth of greater than $20 \times$. Unique sequence reads were aligned with reference genome (GRCh37/hg19) to obtain variant files. The variants were filtered by comparing with NCBI dbSNP database, ExAC database and 1000 Genome database to exclude known polymorphisms with a frequency of more than $5 \%$ in normal subjects, and by searching the Human Gene Mutation Database (HGMD, http://www.hgmd.org/) to clarify whether the variant is a known pathogenic variant. The nomenclature of new variants was based on the international gene variant nomenclature system (http://www.hgvs.org/mutnomen ).

Validation tests of Sanger sequencing. Gene tool software was used for designing primers for suspected variants. Routine PCR reactions were performed. PCR products were purified and directly sequenced on $\mathrm{AB} 13130-\mathrm{xl}$ gene sequencing instrument using the ABIBigDye3.1 sequencing kit (Thermo Fisher Scientific, USA), and the sequencing data were compared and analyzed using ABI Sequencing Analysis 5.1.1 software.

In silico webservers and structure prediction. Multiple sequence alignments were performed using HomoloGene database (http://www.ncbi.nlm.nih.gov/homologene) to verify the degree of conservation. The pathogenicity of the variants was then evaluated using three in silico webservers, PolyPhen2, SIFT and Mutation Taster2.The American College of Medical Genetics and Genomics(ACMG) guideline was applied to assess novel variants' pathogenicity. Computational modeling was carried out to observe the effect of new missense mutations on protein structure. Three-dimensional structure of the target protein sequence was constructed using PyMOL protein 
model structure simulation software to determine the effect of amino acid substitution on protein structure.

\section{Results}

Molecular analysis in $B C K D H A, B C K D H B$ and $D B T$ genes. NGS was performed in children of families 3 and 7 and couples of the other 6 MSUD families to detect the sequence variation in each exon of the 4 causative genes (BCKDHA, BCDKHB, DBT, DLD) associated with MSUD. After alignment with the hg19 sequence, the variants were filtered by excluding the SNPs (normal frequency $>0.05$ ) reported in the dbSNP137 database, Hapmap database and 1000 Genome database. Suspected variants in the causative genes associated with the 8 families were shown in Table 2.

Sanger sequencing results. The suspected variants found by NGS were confirmed by Sanger sequencing. The patient in family 3 carried $B C K D H B$ gene c.511T $>\mathrm{C}(\mathrm{p}$. Ser171Pro) and $\mathrm{c}$. c.547C $>\mathrm{T}$ (p.Arg183Trp) compound heterozygous variants, and the children in pedigree 7 carried c.508C $>$ T(p.Arg170Cys) and c.478-552del(p.I160Ffs*25) compound heterozygous variants. Their parents were heterozygous carriers of the respective variant. Heterozygous variants in the same causative gene of MSUD were detected in both couples in the remaining six families.Gene sequences of four novel variant in $B C K D H A$ and $B C K D H B$ genes were shown in Figure 1.

A

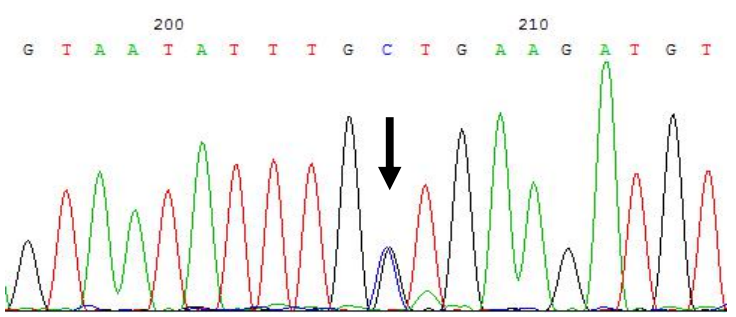

$\mathrm{C}$

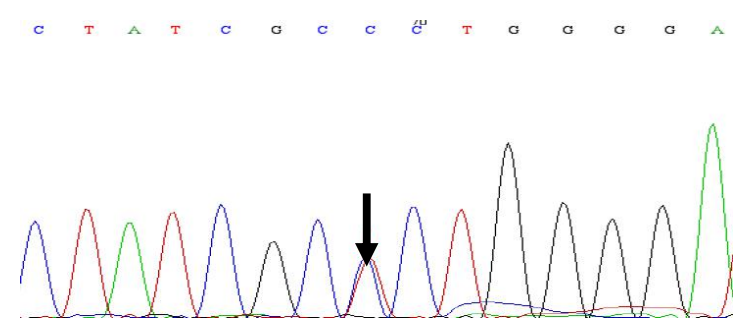

$\mathrm{B}$

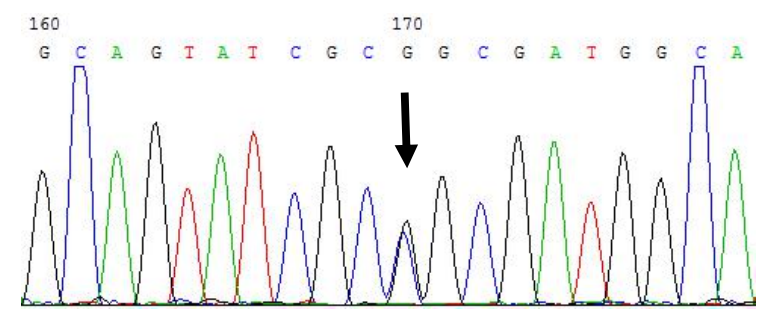

$\mathrm{D}$

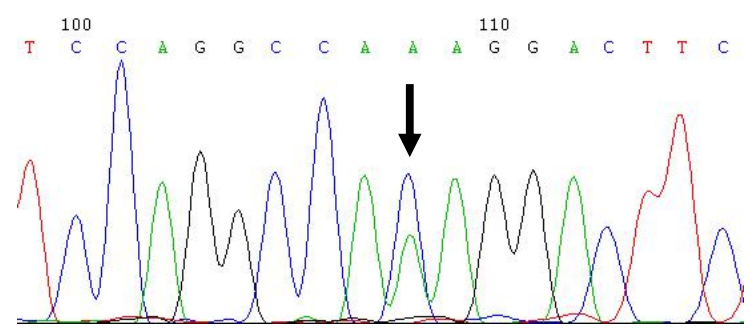

Figure 1: Gene sequences of four novel variant in $B C K D H A$ and $B C K D H B$ genes in eight pedigrees: c.841G $>\mathrm{C}($ p.Gly281Arg) in $B C K D H A$ gene(A), c.284G $>\mathrm{C}($ p.Gly95Ala) in $B C K D H B$ gene(B), c.511T $>$ C(p.Ser171Pro) in $B C K D H B$ gene(C), c.665A $>C$ (p.Lys222Thr) in $B C K D H B$ 
gene (D)

In silico prediction of novel gene variant sequences. Mutation Taster and PolyPhen-2 analysis showed that four novel missense variants:p.Gly95Ala, p.Gly281Arg, p.Lys222Thr and p.Ser171Pro were highly likely to be pathogenic/deleterious variants. We use The American College of Medical Genetics and Genomics(ACMG) guideline to assess these novel variants' pathogenicity in Table 2.

Table 2:Detection and analysis of gene mutation in 8 MSUD families

\begin{tabular}{|c|c|c|c|c|c|c|}
\hline \multirow{2}{*}{ Pedigree } & \multirow{2}{*}{ Gene } & \multicolumn{2}{|c|}{ Proband genotype } & \multirow{2}{*}{$\begin{array}{l}\text { Paternal } \\
\text { genotype }\end{array}$} & \multirow{2}{*}{$\begin{array}{l}\text { Maternal } \\
\text { genotype }\end{array}$} & \multirow{2}{*}{$\begin{array}{l}\text { Pathogenicity assessment } \\
\text { based on ACMG guideline }\end{array}$} \\
\hline & & Genotype 1 & Genotype 2 & & & \\
\hline 1 & $B C K D H A$ & / & / & p.Gly281 Arg & p.Gly281Arg & PM3+PP3 \\
\hline 2 & $B C K D H A$ & / & / & p.Ala216Val & p.Ala216Val & \\
\hline 3 & $B C K D H B$ & p.Ser171Pro & p.Arg183Trp & p.Arg183Trp & p.Ser171Pro ${ }^{\#}$ & PM3+PP3 \\
\hline 4 & $B C K D H B$ & I & I & p.Phe175Leu & p.Lys222Thr ${ }^{\#}$ & PM3+PM5+PP3 \\
\hline 5 & $B C K D H B$ & / & l & p.Gly95Ala & p.Arg285Ter & PM3+PP3 \\
\hline 6 & BCKDHB & / & / & p.Arg111Ter & p.S184Pfs*46 & \\
\hline 7 & $B C K D H B$ & p.Arg170Cys & p.I160Ffs $* 25$ & p.Arg170Cys & p.I160Ffs $* 25$ & \\
\hline 8 & $D B T$ & l & l & p.Arg431Ter & p.Arg431Ter & \\
\hline
\end{tabular}

Notes: "novel variation

Three-dimensional structure of proteins. The predicted three-dimensional structures of 4 novel variants in $B C K D H A$ and $B C K D H B$ genes were shown in Fig.2. In $B C K D H A$ gene, Glycine 281 is located in the random coil structure of protein secondary structure. Glycine lacks side chain (only one H-bond). After mutation to arginine, arginine is a basic amino acid with ions, affecting the stability of E1 $\alpha$ tertiary structure, thus affecting protein function.

In $B C K D H B$ gene, As is shown in Fig.2F, Serine at position 171 is located in the a -helix of protein secondary structure, forming hydrogen bonds with Leucine at position 174, Phenylalanine at position 175 and Tyrosine at position 167. After mutation to Proline, the hydrogen bonds with Leucine at position 167 and Phenylalanine at position 175 disappear, affecting the stability of protein secondary structure. Therefore, it is speculated that $\mathrm{p}$.Ser171Pro mutation has a greater impact on protein function.

Amino acid 222 is located in the $\alpha$-helix of the secondary structure of the protein, amino acid 222 forms hydrogen bonds with amino acids 79, 83, 218, 225, 252, and 254.After mutation to Threonine, it reforms hydrogen bonds with amino acids 76,218 , and 225.The secondary structure of the protein is changed, which disrupts the stability of the protein and may affect the cleavage 
and activation function of the protein.

Gly95 is located in the $\beta$-turn region, and due to the lack of side chains (only one $\mathrm{H}$ atom) by Glycine, there is no steric hindrance, allowing a U-shaped turn of the peptide chain by $180^{\circ}$ in the $\beta$-turn. Mutation of Glycine to Alanine, which forms some steric hindrance, is likely to change the turning of the $\beta$-turn, causing change in local steric conformation.

A

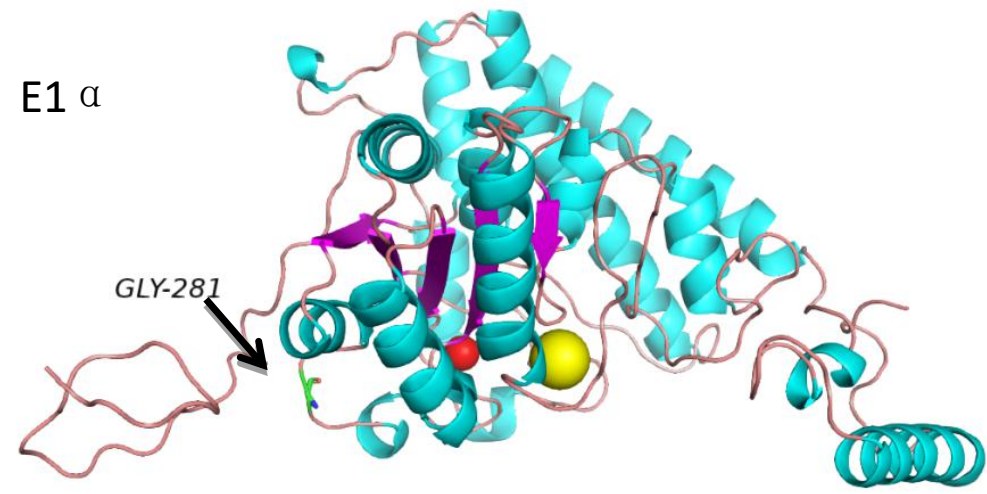

Fig.2.(A):Predicted

$3 \mathrm{D}$ protein structure of

E1 $\alpha$ compotent complex and the site of the Gly cine(Gly281) colored in green

B

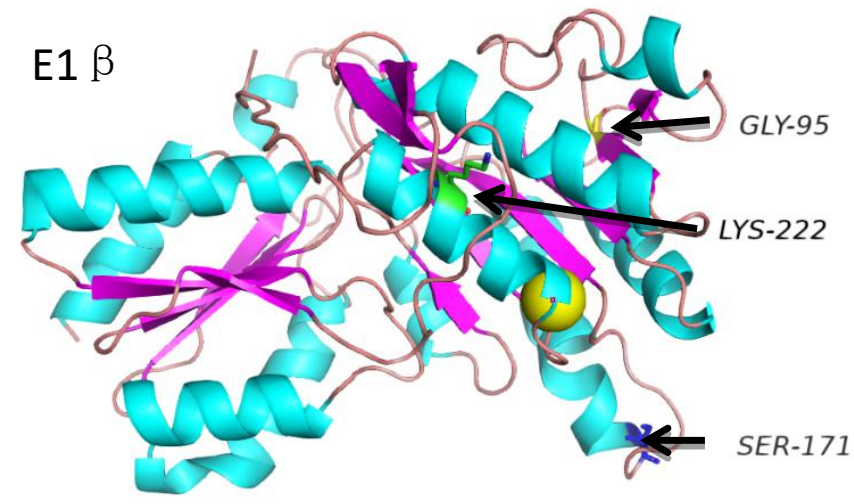

Fig.2.(B): Predicted 3D protein structure of E1 $\beta$ compotent complex and the site of three residues. The Glycine(Gly 95),Lysine(Lys222),Serin e(Ser171) are colored yellow,green and blue respectively. 
$\mathrm{C}$

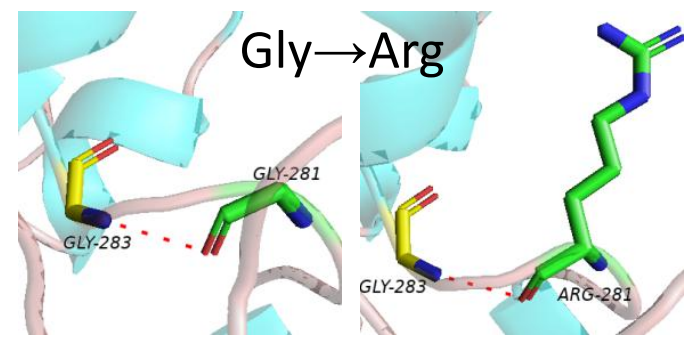

$\mathrm{E}$

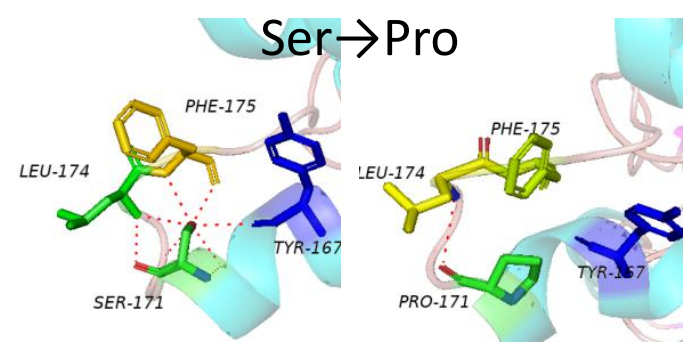

$\mathrm{D}$

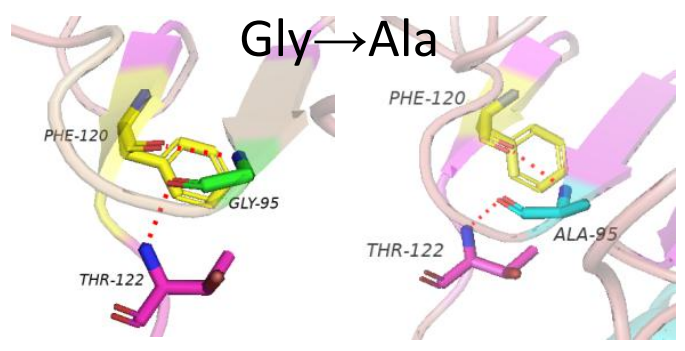

$\mathrm{F}$

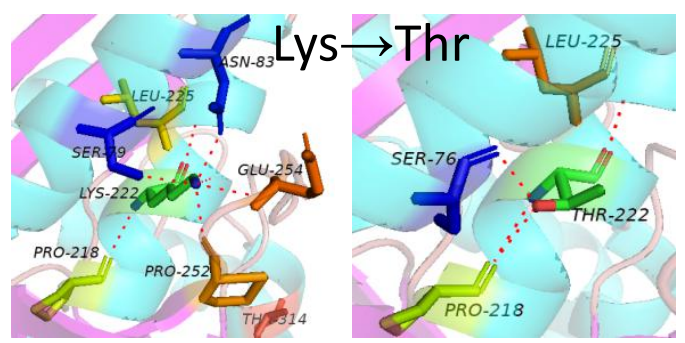

Figure 2: Predicted protein structures of four novel variants in $B C K D H A$ and $B C K D H B$ genes: p.Gly281 $\operatorname{Arg}(\mathrm{C})$ in $B C K D H A$ gene, p.Gly95Ala(D), p.Ser171Pro(E) and p.Lys222Thr(F) in $B C K D H B$ gene

\section{Discussion}

Maple syrup urine diabetes is a branched-chain amino acid metabolism disease caused by mitochondrial branched-chain $\alpha$-keto acid dehydrogenase (BCKDC) deficiency. Scaini et al. ${ }^{9}$ suggested that cognitive impairment after accumulation of branched-chain amino acids is mainly due to oxidative damage to the brain. The clinical manifestations of MSUD are lack of specificity with rapid onset. The detection of amino acid levels and the ratio between related amino acids in hemofilter paper by tandem mass spectrometry ${ }^{10}$ allow for early screening of MSUD and provide an important basis for further diagnosis and treatment. In this study, the results of blood tandem mass spectrometry in all families showed that both leucine and valine were significantly higher, accompanied by amino acid ratio changes, consistent with MSUD biochemical findings.

Gene mutations of MSUD patients are mainly concentrated in the $B C K D H B$ gene, followed by $B C K D H A$ and $D B T$ genes $^{11}$. Current studies suggest that patients with $B C K D H A$ and $B C K D H B$ gene mutations mostly present with classical type, $\mathrm{BCKDH}$ activity is less than $2 \%$. $D B T$ gene variants accounts for about $24 \%$, and most of them are thiamine effective type ${ }^{12}$. The clinical manifestations of patients are relatively mild, including developmental retardation and hypotonia. 
$D L D$ gene variants account for $13 \%{ }^{13}$. Each gene has high allelic heterogeneity, with the exception of gene mutation hotspots found in minority of ethnic groups, such as the most common mutation in the Mennonite community being the BCKDHA gene c.1312T >A (p.Tyr393Asn) ${ }^{14}$, Portuguese gypsy mutation hotspot c. $117 \mathrm{delC}^{15}$. The $B C K D H B$ gene $\mathrm{c} .538 \mathrm{G}>\mathrm{C}$ was a common mutation found in Ashkenazi Jews ${ }^{16}$, and exon 5 of the $B C K D H B$ gene may be a region of genetic variation and a hotspot region ${ }^{17,18}$. Hotspot mutations are not found in the remaining population ${ }^{19-21}$. There were few reports on MSUD gene mutations in the Chinese population ${ }^{22-24}$, and no significant hotspot mutations have been identified. In this study, a total of 13 gene variants $(15.4 \%$ located in $B C K D H A$ gene, $76.9 \%$ in $B C K D H B$ gene, and $7.7 \%$ in $D B T$ gene) were found in 16 alleles in 8 families, and the mutation frequency of the $B C K D H B$ gene was the highest, which was consistent with the results of other studies ${ }^{25-27}$. The $B C K D H B$ gene may be a major variant type of MSUD in the Chinese population. Four of these were novel variants, one located in $B C K D H A$ gene p. Gly281Arg and three located in $B C K D H B$ gene p. Gly95Ala, p.Lys222Thr, p.Ser171Pro illustrates that the disease has high allelic heterogeneity.

The severity of the MSUD metabolic phenotype is determined by the amount of residual BCKD enzyme activity. The relationship between MSUD genotype and phenotype has not yet been established. The incidence of the disease is low, and fewer cases are included in each study, making it difficult to obtain an exact genotype-phenotype relationship. Patients with nonsense mutations presented the severe classic phenotype. Mutations in p.Arg111Ter and p.Arg285Ter in $B C K D H B$ gene generate premature termination codons and the encoded protein has serious effects on the activity of the complex ${ }^{28}$. Our cases in family 5 and 6 carry the nonsense mutation p.Arg111Ter and p.Arg285Ter, respectively, and they have classic phenotype. However, the same type of genetic variation leads to different clinical phenotypes. For example, In $B C K D H A$ gene, p.Glu327Lys has been reported to be associated with intermediate phenotype ${ }^{8}$ while the same mutation results in classic phenotype in patients in another study ${ }^{18}$. In our study, patient 3 carries the missense mutation p.Arg183Trp in the $B C K D H B$ gene and show intermediate phenotype, while the patient who had the same mutation showed classic phenotype in a previous report ${ }^{29}$. Therefore, we could not establish any genotype-phenotype correlation in our patients with MSUD. Half of our cases are classical phenotypes and half are intermediate phenotypes. Majority of 
patients with intermediate phenotype had mutations in the $B C K D H B$ gene. All the three genes are implicated in classic phenotype. All patients with classic phenotype have worst clinical outcome.

MSUD is a fatal and disabling inherited metabolic disease which is difficult to treat, and has a poor prognosis. Untreated classical children mostly die shortly after birth ${ }^{29}$. Currently, the best preventive strategy for the disease is to avoid the birth of affected children through prenatal diagnosis. However, at the time of presentation of the disease family in clinic, the proband may have died, and novel genetic variants that may cause the disease could be found in the parents of the family. When prenatal diagnosis was performed in the next pregnancy of the same family, pretest counseling should be carefully performed regarding the limitations of result interpretation and prognosis prediction, and informed consent should be obtained before further investigation. Additionally, the traditional prenatal diagnosis method by chorionic villi sampling, amniocentesis and cordocentesis has a certain risk of miscarriage. Although non-invasive genetic diagnosis through haplotype linkage analysis has been developed to assist early diagnosis of MSUD ${ }^{30}$, technical and logistical problems must be worked out before the strategy can be clinically useful for MSUD. Preimplantation genetic testing for mendelian disorders (PGT-M) is another prevention strategy for avoiding birth of affected children. However, the current method of PGT-M requires the specimen of the proband for linkage analysis, while the specimen of children with MSUD cannot be obtained because of early death, which greatly limits the clinical application of PGT-M. By contrast, neonatal screening is the key to early diagnosis, and striving for the timing of treatment is an important factor to improve the prognosis of maple syrup diabetes $^{31}$. When MUSD is clinically suspected, capture-based high throughput sequencing followed by Sanger sequencing confirmation allows for accurate detection of gene mutations in the causative genes in an effective manner.

In summary, we applied high-throughput sequencing technology based on target gene capture for sequencing of the four genes in the 8 families with MSUD. NGS combined with Sanger sequencing can detect gene mutations in the causative genes in an effective way, providing clinicians with the basis for differential diagnosis, drug treatment, subsequent genetic counseling and prenatal diagnosis.

Acknowledgements We would like to thank our colleagues. Without their referrals this would be incomplete. Last but not least we would like to thank the patients and their families for their 
sacrifices and giving us their precious blood samples and clinical information. All authors reviewed the manuscript.

Funding This work was supported financially by National Natural Science Foundation of China (81701533, 81672110) for which we are grateful.

Competing interests The author(s) declare no competing interests.

\section{References:}

1 Miryounesi, M., Ghafouri-Fard, S., Goodarzi, H. \& Fardaei, M., A new missense mutation in the BCKDHB gene causes the classic form of maple syrup urine disease (MSUD). $J$ Pediatr Endocrinol Metab 28673 (2015).

2 Couce, M. L. et al., Evolution of maple syrup urine disease in patients diagnosed by newborn screening versus late diagnosis. Eur J Paediatr Neurol 19652 (2015).

3 Pode-Shakked, N. et al., Clues and challenges in the diagnosis of intermittent maple syrup urine disease. EUR J MED GENET 63103901 (2020).

4 Axler, O. \& Holmquist, P., Intermittent maple syrup urine disease: two case reports. PEDIATRICS 133 e458 (2014).

5 Scriver, C. R., Clow, C. L. \& George, H., So-called thiamin-responsive maple syrup urine disease: 15-year follow-up of the original patient. The Journal of pediatrics 107763 (1985).

6 Mitsubuchi, H., Owada, M. \& Endo, F., Markers associated with inborn errors of metabolism of branched-chain amino acids and their relevance to upper levels of intake in healthy people: an implication from clinical and molecular investigations on maple syrup urine disease. J NUTR 135 $1565 \mathrm{~S}$ (2005).

7 Nellis, M. M. et al., Relationship of causative genetic mutations in maple syrup urine disease with their clinical expression. MOL GENET METAB 80189 (2003).

8 Rodriguez-Pombo, P., Navarrete, R., Merinero, B., Gomez-Puertas, P. \& Ugarte, M., Mutational spectrum of maple syrup urine disease in Spain. HUM MUTAT 27715 (2006).

9 Scaini, G. et al., Antioxidant administration prevents memory impairment in an animal model of maple syrup urine disease. BEHAV BRAIN RES 23192 (2012).

10 Chace, D. H. et al., Rapid diagnosis of maple syrup urine disease in blood spots from newborns by tandem mass spectrometry. CLIN CHEM 4162 (1995).

11 Ali, E. Z. \& Ngu, L., Fourteen new mutations of BCKDHA, BCKDHB and DBT genes associated with maple syrup urine disease (MSUD) in Malaysian population. Molecular Genetics and Metabolism Reports 1722 (2018).

12 Chuang, D. T., Chuang, J. L. \& Wynn, R. M., Lessons from genetic disorders of branched-chain amino acid metabolism. J NUTR 136 243S (2006).

13 Georgiou, T. et al., Maple syrup urine disease in Cypriot families: identification of three novel mutations and biochemical characterization of the p.Thr211Met mutation in the E1alpha subunit. Genet Test Mol Biomarkers 13657 (2009).

14 Love-Gregory, L. D., Grasela, J., Hillman, R. E. \& Phillips, C. L., Evidence of common ancestry for the maple syrup urine disease (MSUD) Y438N allele in non-Mennonite MSUD patients. $M O L$ GENET METAB 7579 (2002). 
15 Quental, S. et al., Revisiting MSUD in Portuguese Gypsies: evidence for a founder mutation and for a mutational hotspot within the BCKDHA gene. ANN HUM GENET 73298 (2009).

16 Edelmann, L. et al., Maple syrup urine disease: identification and carrier-frequency determination of a novel founder mutation in the Ashkenazi Jewish population. AM J HUM GENET 69863 (2001).

$17 \mathrm{Su}, \mathrm{L}$. et al., Two homozygous mutations in the exon 5 of BCKDHB gene that may cause the classic form of maple syrup urine disease. METAB BRAIN DIS 32765 (2017).

18 Gupta, D. et al., Identification of mutations, genotype-phenotype correlation and prenatal diagnosis of maple syrup urine disease in Indian patients. EUR J MED GENET 58471 (2015).

19 Abiri, M. et al., Identification of six novel mutations in Iranian patients with maple syrup urine disease and their in silico analysis. Mutat Res 78634 (2016).

20 Imtiaz, F. et al., Twenty novel mutations in BCKDHA, BCKDHB and DBT genes in a cohort of 52 Saudi Arabian patients with maple syrup urine disease. Mol Genet Metab Rep 1117 (2017).

21 Xiyuan Li, et al. Clinical, biochemical and genetic features of 13 children with maple syrup urine disease. Chinese Journal of Applied Clinical Pediatrics. 31, 569-572 (2016).

22 Yang, N. et al., Analysis of gene mutations in Chinese patients with maple syrup urine disease. MOL GENET METAB 106412 (2012).

$23 \mathrm{Li}, \mathrm{X}$. et al., Eleven novel mutations of the BCKDHA, BCKDHB and DBT genes associated with maple syrup urine disease in the Chinese population: Report on eight cases. EUR J MED GENET 58 617 (2015).

$24 \mathrm{Li}, \mathrm{W}$. et al., Silico analysis of a novel mutation c.550delT in a Chinese patient with maple syrup urine disease. Clin Case Rep 61989 (2018).

25 Yang, C., Linpeng, S., Cao, Y. \& Wu, L., Identification of six novel mutations in five infants with suspected maple syrup urine disease based on blood and urine metabolism screening. GENE 7109 (2019).

$26 \mathrm{Li}, \mathrm{X}$. et al., Clinical characteristics and mutation analysis of five Chinese patients with maple syrup urine disease. METAB BRAIN DIS 33741 (2018).

27 Sun, W. et al., Identification of eight novel mutations in 11 Chinese patients with maple syrup urine disease. WORLD J PEDIATR (2020).

28 Flaschker, N. et al., Description of the mutations in 15 subjects with variant forms of maple syrup urine disease. J INHERIT METAB DIS 30903 (2007).

29 Abiri, M. et al., Maple syrup urine disease mutation spectrum in a cohort of 40 consanguineous patients and insilico analysis of novel mutations. METAB BRAIN DIS 341145 (2019).

30 You, Y. et al., Integration of targeted sequencing and NIPT into clinical practice in a Chinese family with maple syrup urine disease. GENET MED 16594 (2014).

31 Strauss, K. A. et al., Classical maple syrup urine disease and brain development: principles of management and formula design. MOL GENET METAB 99333 (2010). 
Figures

A

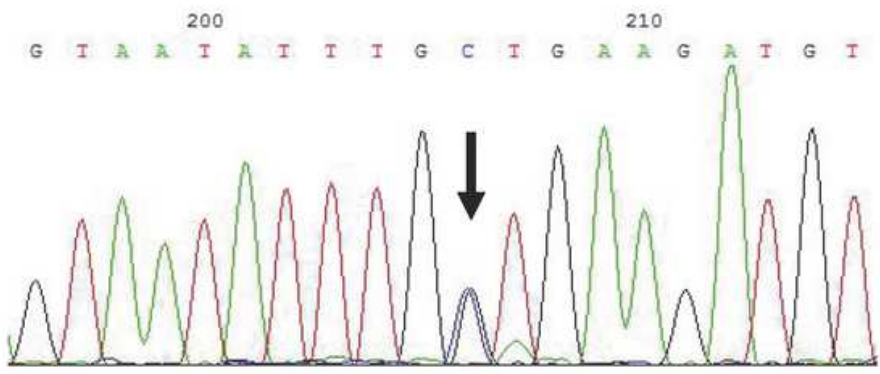

$\mathrm{C}$
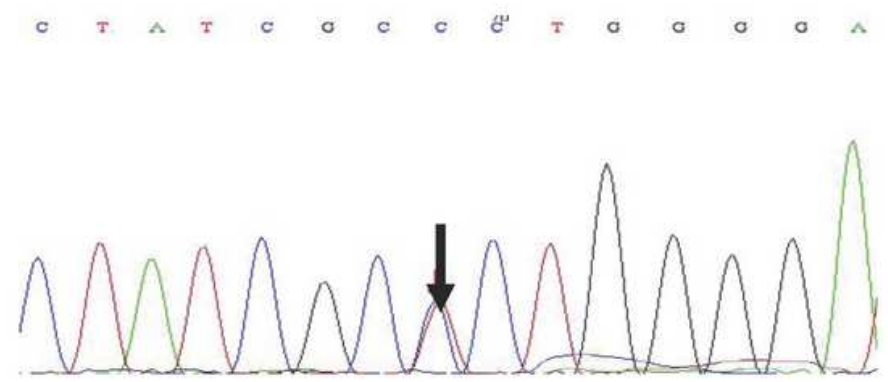

B

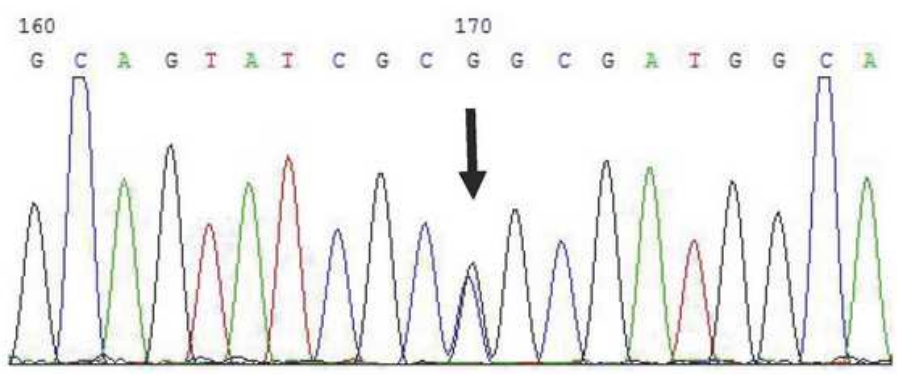

D

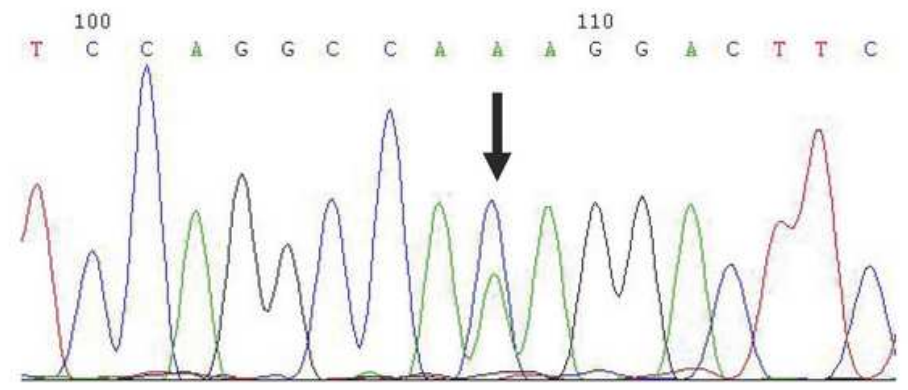

\section{Figure 1}

Gene sequences of four novel variant in BCKDHA and BCKDHB genes in eight pedigrees: c.841G >C(p.Gly281Arg) in BCKDHA gene(A), c.284G>C(p.Gly95Ala) in BCKDHB gene(B), c.511T >C(p.Ser171Pro) in BCKDHB gene(C), c.665A>C(p.Lys222Thr) in BCKDHB gene (D) 
A

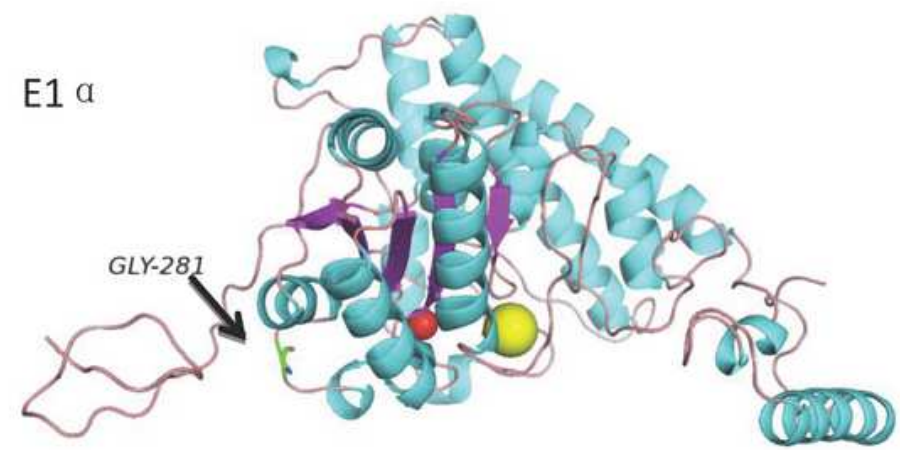

Fig.2.(A):Predicted

3D protein structure of

E1 $\alpha$ compotent complex and the site of the Gly cine(Gly281) colored in green

B

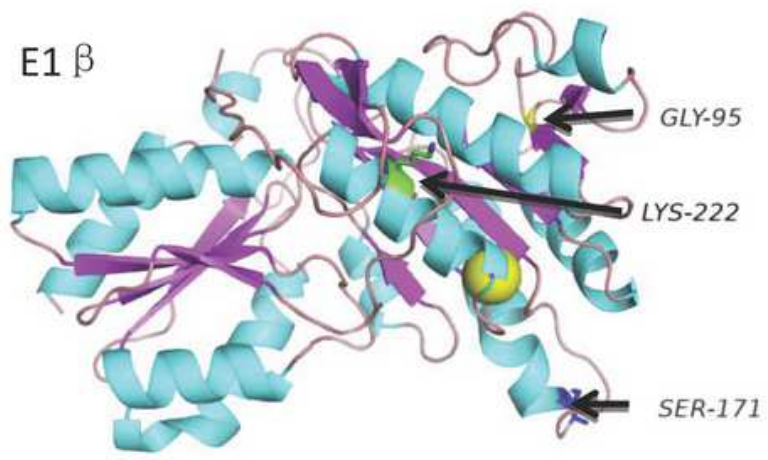

Fig.2.(B): Predicted

3D protein structure of

E1 $\beta$ compotent complex and the site of three residues.The Glycine(Gly 95),Lysine(Lys222), Serin $\mathrm{e}($ Ser171) are colored yellow,green and blue respectively.

C

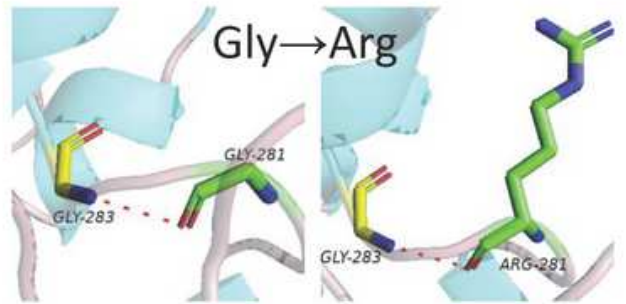

D

E
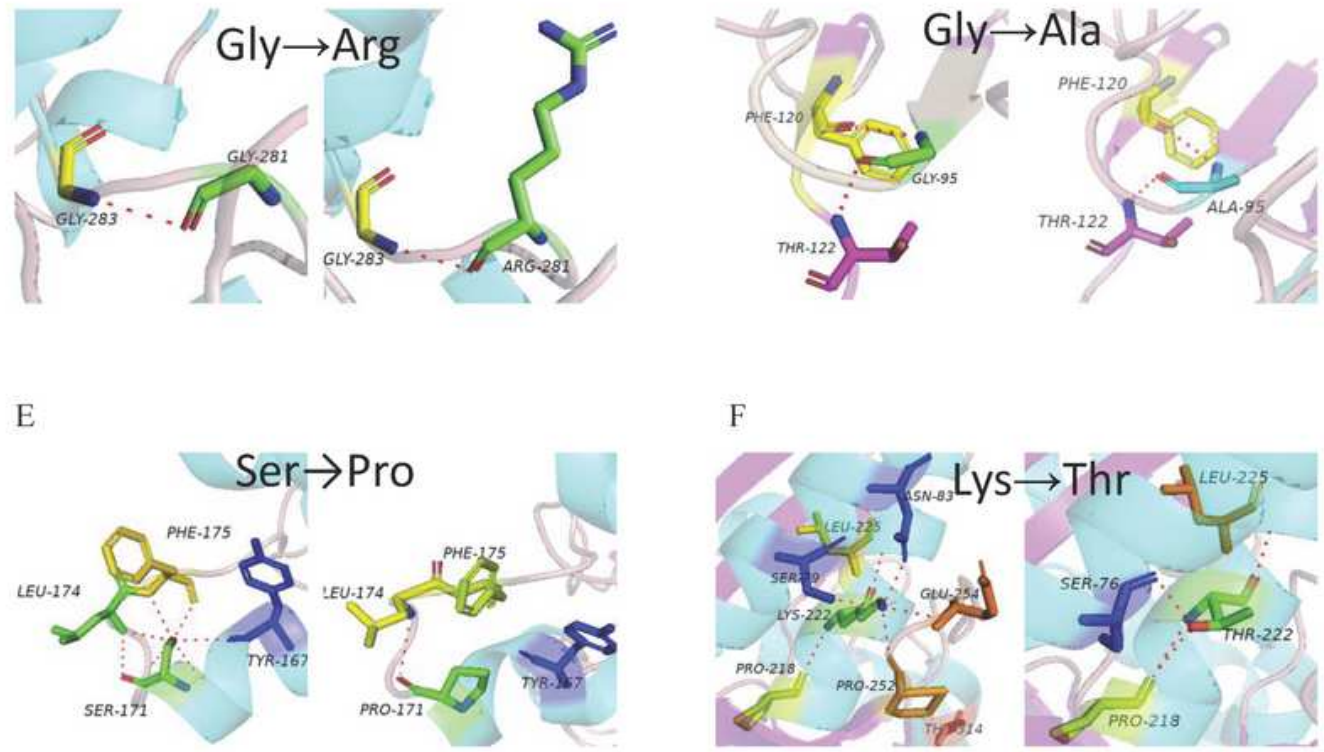

\section{Figure 2}

Predicted protein structures of four novel variants in BCKDHA and BCKDHB genes: p.Gly281 $\operatorname{Arg}(C)$ in BCKDHA gene, p.Gly95Ala(D), p.Ser171Pro(E) and p.Lys222Thr(F) in BCKDHB gene

\section{Supplementary Files}


This is a list of supplementary files associated with this preprint. Click to download.

- Table.pdf 